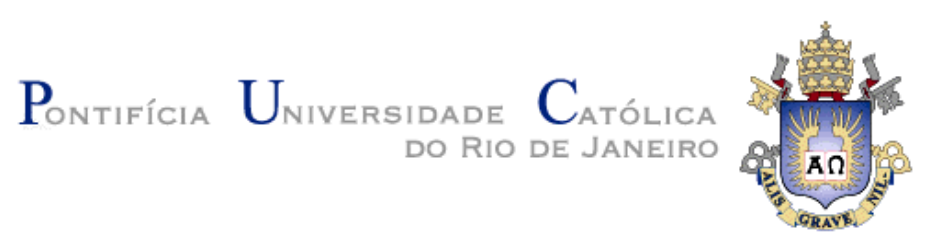

Débora Dias Jardim Penna

\title{
Definição da Árvore de Cenários de Afluências para o Planejamento da Operação Energética de Médio Prazo
}

Tese de Doutorado

Tese apresentada como requisito parcial para obtenção do título de Doutor pelo Programa de PósGraduação em Engenharia Elétrica da PUC-Rio.

Orientador: Reinaldo Castro Souza

Co-Orientadora: Maria Elvira Piñeiro Maceira

Rio de Janeiro

Julho de 2009 


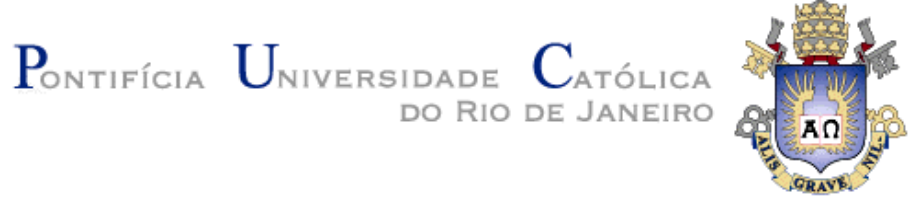

Débora Dias Jardim Penna

\section{Definição da Árvore de Cenários de Afluências para o Planejamento da Operação Energética de Médio Prazo}

Tese de Doutorado apresentada como requisito parcial para obtenção do título de Doutor pelo Programa de PósGraduação em Engenharia Elétrica do Departamento de Engenharia Elétrica do Centro Técnico Científico da PUC-Rio. Aprovada pela Comissão Examinadora abaixo assinada.

Dr. Reinaldo Castro Souza Orientador Departamento de Engenharia Elétrica/PUC-Rio

Dra. Maria Elvira Piñeiro Maceira Co-Orientadora UERJ/CEPEL

Dra. Marley Maria Bernardes Rebuzzi Vellasco Departamento de Engenharia Elétrica /PUC-Rio

Dr. Ricardo Tanscheit Departamento de Engenharia Elétrica /PUC-Rio

Dr. Jorge Machado Damázio UERJ/CEPEL

Dr. Paulo Roberto de Holanda Sales UERJ/ELETROBRAS

Prof. José Eugenio Leal Coordenador Setorial do Centro

Técnico Científico - PUC-Rio

Rio de Janeiro, 09 de julho de 2009 
Todos os direitos reservados. É proibida a reprodução total ou parcial do trabalho sem autorização da universidade, da autora e do orientador.

\section{Débora Dias Jardim Penna}

Graduou-se em Engenharia Elétrica pela Universidade Federal de Juiz de Fora, UFJF (1997). Mestre em Ciênicas em Engenharia Elétrica pela Universidade Federal do Rio de Janeiro, UFRJ (2002). Desde 1998 é pesquisadora do Centro de Pesquisas de Energia Elétrica, CEPEL, onde tem participado do desenvolvimento de modelos e sistemas computacionais para planejamento da operação energética de sistemas hidrotérmicos interligados e geração de cenários sintéticos multivariados de vazões e energias.

Ficha Catalográfica

Penna, Débora Dias Jardim

Definição da árvore de cenários de afluências para o planejamento da operação energética de médio prazo / Débora Dias Jardim Penna ; orientador: Reinaldo Castro Souza ; co-orientadora: Maria Elvira Piñeiro Maceira. 2009.

$293 \mathrm{f.} ; 30 \mathrm{~cm}$

Tese (Doutorado em Engenharia Elétrica)-Pontifícia Universidade Católica do Rio de Janeiro, Rio de Janeiro, 2009.

Inclui bibliografia

1. Engenharia elétrica - Teses. 2. Planejamento da operação energética. 2. Geração de séries sintéticas multivariadas. 3. Geração de árvore de cenários. 4. Técnicas de agregação. 5. Técnicas de amostragem. I. Souza, Reinaldo Castro. II. Piñeiro Maceira, Maria Elvira. III. Pontifícia Universidade Católica do Rio de Janeiro. Departamento de Engenharia Elétrica. IV. Título. 


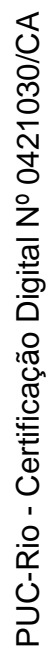

Para meu querido vô Moacir

(in memorian) 


\section{Agradecimentos}

À orientadora Maria Elvira Maceira pela excelente orientação ao longo deste trabalho. Agradeço o voto de confiança e a oportunidade de trabalharmos no mesmo grupo de pesquisa.

Ao professor Reinaldo Souza, por ter proporcionado o intercâmbio PUC/CEPEL, sem o qual este trabalho não teria sido realizado.

Ao pesquisador Jorge Damázio pelas discussões e observações fundamentadas na sua sólida experiência.

Aos demais membros da banca, Marley Vellasco, Ricardo Tanscheit e Paulo Holanda pelas valiosas contribuições ao texto final da tese.

Ao Centro de Pesquisas em Energia Elétrica (CEPEL), na figura de seu diretor Albert Melo, pelo grande apoio e pela oportunidade de desenvolvimento deste trabalho.

Aos amigos Vitor Duarte, Welington Oliveira e André Diniz pelas enriquecedoras discussões que muito me auxiliaram neste trabalho.

Ao pesquisador Roberto Pinto, pela grande ajuda com o universo Linux.

Aos amigos Luiz Guilherme e Fábio Batista pelo incentivo constante e pelos boletins diários da tese. Aos demais amigos do CEPEL pelo apoio e pela amizade incondicional oferecida.

Aos engenheiros do Operador Nacional do Sistema Elétrico (ONS) Alberto Kligerman, Cecília Mércio, Maria Cândida Lima, Maria Helena de Azevedo, Joari Costa e Murilo Soares pelas importantes discussões travadas.

Aos professores e funcionários do Departamento de Engenharia Elétrica da PUC-Rio, pelo excelente trabalho que realizam.

À PUC-Rio, pelo suporte financeiro.

Ao meu marido Leandro e meu filho Pedro por serem minha fonte de força e energia, e pelo apoio nos momentos mais difíceis dessa longa trajetória.

À minha família que sempre esteve ao meu lado, acreditando e torcendo por mim. Em especial, à vó Cida pelo exemplo de vida.

Finalmente, a Deus, por ter me dado a benção da vida, assim como os melhores presentes: a minha família e os meus amigos. 


\section{Resumo}

Penna, Débora Dias Jardim; Castro, Reinaldo Souza. Definição da Árvore de Cenários de Afluências para o Planejamento da Operação Energética de Médio Prazo. Rio de Janeiro, 2009. 293p. Tese de Doutorado - Departamento de Engenharia Elétrica, Pontifícia Universidade Católica do Rio de Janeiro.

No modelo atualmente utilizado para o planejamento da operação de médio prazo do Sistema Interligado Nacional, a incerteza referente às afluências é considerada explicitamente no cálculo de valores da função de custo futuro bem como ao se percorrer o espaço de estados através da utilização de cenários hidrológicos multivariados. O conjunto de todas as possíveis realizações do processo estocástico de afluências, ao longo de todo horizonte de planejamento, forma uma árvore de cenários. Esta árvore representa todo o universo probabilístico sobre o qual é efetuado o processo de otimização da operação energética. Como a árvore possui uma cardinalidade bastante elevada, torna-se impossível do ponto de vista computacional percorrer completamente a árvore. Portanto, apenas uma porção da árvore (sub-árvore) é percorrida. Atualmente a sub-árvore é definida utilizando amostragem aleatória simples. Este trabalho tem o objetivo de propor um método para a definição da sub-árvore a ser visitada durante o processo do cálculo da política ótima de operação por programação estocástica dual com o intuito de tornar mais robusto os resultados obtidos por esta política de operação em relação a variações no número de cenários das simulações forward e backward e em relação a variações da amostra de cenários hidrológicos utilizada. Duas propostas são aplicadas na definição da sub-árvore: (i) utilizar a amostragem por hipercubo latino ou amostragem descritiva no modelo de geração de cenários hidrológicos multivariados, e (ii) aplicar técnicas estatísticas multivariadas capazes de agrupar objetos similares em determinados grupos (técnicas de agregação). Estas propostas podem ser aplicadas separadamente ou em conjunto.

\section{Palavras-chave}

Planejamento da operação energética; geração de séries sintéticas multivariadas; geração de árvore de cenários; técnicas de agregação; técnicas de amostragem 


\section{Abstract}

Penna, Débora Dias Jardim; Castro, Reinaldo Souza (Advisor). Definition of the Streamflow Scenario Tree to Long-Term Operation Planning. Rio de Janeiro, 2009. 293p. D.Sc. Thesis - Departamento de Engenharia Elétrica, Pontifícia Universidade Católica do Rio de Janeiro.

In the planning operation the currently used model in the long-term operation planning of the Brazilian Interconnected System, the uncertainty concerning streamflow is considered explicitly in the estimation of the expected cost-to-go function, as well as in the covering of the state space, by the use of multivariate hydrological scenarios. The set of all possible realizations of the streamflow stochastic process throughout the planning horizon forms a scenario tree. This tree represents the entire probabilistic universe on which are calculated the optimal operation strategies. As the scenario tree of the long-term operation planning problem has a high cardinality, makes it impossible to visit the complete tree due to computational effort. Therefore, only a portion of the tree (sub-tree) is covered. Currently the sub-tree is selected using the Monte-Carlo method with classical simple random sampling. The objective of this work is to propose a method for defining the sub-tree to be visited during the calculation of the optimal operating strategy for the Brazilian hydro-thermal power system by stochastic dual dynamic programming in order to obtain more robust results from this operation policy with regard to variations in the number of scenarios of forward and backward simulations, and variations in the sample hydrological scenario. There are two proposals for definition of the sub-tree: (i) change the simple random sampling to latin hypercube sampling or descriptive sampling in the multivariate streamflow scenario generation model, and (ii) apply multivariate statistical techniques to develop criteria that allow grouping similar objects in certain groups (clustering techniques). The proposals can be applied together or separately.

\section{Keywords}

long-term operation planning; multivariate synthetic scenarios generation; scenarios tree generation; clustering techniques; sampling techniques 


\section{Sumário}

1 Introdução 23

1.1. Considerações Iniciais 23

1.2. Contexto do Trabalho 25

1.3. Objetivos do Trabalho 26

$\begin{array}{ll}\text { 1.4. Metodologia Proposta } & 27\end{array}$

1.5. Relevância do Trabalho $\quad 27$

1.6. Organização do Trabalho 28

2 Planejamento da Operação Energética 30

2.1. Considerações Iniciais 30

2.2. Formulação do Problema 31

2.2.1. Sistemas Térmicos 31

2.2.2. Sistemas Hidrotérmicos 32

2.3. Planejamento da Operação Energética no Sistema Brasileiro 35

2.3.1. Cadeia de Modelos 37

2.3.2. Planejamento de Médio Prazo 38

2.3.3. Planejamento de Curto Prazo 40

2.3.4. Programação da Operação 40

2.4. Resumo 41

3 Planejamento da Operação de Médio Prazo 42

3.1. Considerações Iniciais 42

3.2. Representação da Incerteza Hidrológica na PDDE 43

3.3. Construção da Árvore de Cenários $\quad 47$

3.4. Resumo 51

4 Modelo de Geração de Séries Sintéticas de Energia 52

4.1. Considerações Iniciais 52

4.2. Processos Estocásticos e Séries Temporais 53

4.3. O Modelo Autorregressivo Periódico 56

4.3.1. Descrição do Modelo 56

4.3.2. Ajuste do Modelo $\quad 59$ 
4.3.3. Geração de Séries Sintéticas com o Modelo PAR(p) 61

4.3.4. Vazões Incrementais Negativas $\quad 65$

4.3.5. Correlação Espacial 66

4.4. Avaliação do Desempenho do Modelo 66

4.4.1. Geração em Paralelo 66

4.4.2. Geração em Árvore $\quad 71$

4.5. Resumo 74

5 Definição da Árvore de Cenários $\quad 75$

5.1. Considerações Iniciais 75

5.2. Técnicas de Seleção de Cenários 75

5.2.1. Métodos de Agrupamento 77

5.2.2. Método K-MEANS 80

5.3. Técnicas de Amostragem 81

5.3.1. Amostragem por Hipercubo Latino $\quad 81$

5.3.2. Amostragem Descritiva $\quad 84$

5.3.3. Quase-Monte Carlo 85

5.4. Resumo $\quad 87$

6 Aplicação do Modelo de Geração de Cenários 88

6.1. Considerações Iniciais 88

6.2. Alternativas para Construção da Árvore de Afluências 88

6.3. Construção dos Cortes de Benders 94

6.4. Reamostragem de Cenários (recombinação de ruídos) 96

6.5. Considerações Finais 99

6.6. Resumo 100

7 Avaliação dos Cenários Hidrológicos Gerados $\quad 101$

7.1. Considerações Iniciais 101

7.2. Análise da amostra de ruídos 102

7.2.1. Amostra de ruídos utilizada no processo de agregação 102

7.2.2. Amostra de ruídos utilizada na geração dos cenários do passo forward 107

7.2.3. Amostra de ruídos utilizada na geração dos cenários do passo

backward 111

7.2.4. Variando o tamanho da amostra de ruídos forward e backward 114

7.2.5. Amostra de ruídos utilizando LHC e AD 122

7.3. Análise dos Cenários Gerados para Passo Forward 125 
7.3.1. Envoltória dos Cenários 126

7.3.2. Média dos Cenários 130

7.3.3. Desvio-padrão dos Cenários 132

7.3.4. Distribuição Univariada dos Cenários 134

7.3.5. Correlação Cruzada dos Cenários 138

7.3.6. Análise de Seqüências Negativas 143

7.4. Análise dos Cenários Gerados para Passo Backward 149

7.4.1. Envoltória dos Cenários 150

7.4.2. Testes não condicionados para média e desvio 155

7.4.3. Distribuição Univariada dos Cenários 157

7.4.4. Testes condicionados para média e desvio 162

7.4.5. Correlação Cruzada dos Cenários 164

7.5. Resumo 172

8 Avaliação dos Resultados do Problema de Planejamento da Operação 174

8.1. Considerações Iniciais 174

8.2. Avaliação da convergência 176

8.3. Alteração da amostra de cenários $\quad 179$

8.4. Alteração do número de cenários backward 183

8.5. Alteração do número de cenários forward 186

8.6. Alteração do tamanho da amostra para o processo de agregação 191

8.7. Alteração do método de amostragem 193

8.8. Combinação de método de amostragem e agregação 197

8.9. Verificação da estabilidade dos resultados 203

8.10. Resumo 213

9 Conclusões e Sugestões para Trabalhos Futuros 214

$\begin{array}{ll}9.1 . & \text { Sugestões para trabalhos futuros } \\ \end{array}$

10 Referências Bibliográficas $\quad 219$

Apêndice A Modelo Newave $\quad 225$

A.1. Introdução 225

A.2. Modelo Newave 226

A.2.1. Módulo de Cálculo do Sistema Equivalente 228

A.2.2. Módulo de Cálculo da Política de Operação 231

A.2.2.1. Programação Dinâmica Dual Estocástica Aplicada ao 
Planejamento da Operação Hidrotérmica

A.2.2.2 Despacho de Operação Hidrotérmica em Sistemas Equivalentes de Energia

A.2.3. Módulo de Simulação da Operação

Apêndice B Análise dos Cenários Gerados de Energia Natural Afluente (Complementação)

B.1 Análise dos Cenários Gerados para Passo Forward 241

B.1.1 Média dos Cenários 241

B.1.2 Desvio-padrão dos Cenários $\quad 245$

B.2. Análise dos Cenários Gerados para Passo Backward 250

B.2.2 Testes não Condicionados para Média e Desvio-padrão 250

B.2.2 Testes Condicionados para Média e Desvio-padrão 255

B.2.3 Testes Condicionados para Correlação Cruzada 259

Apêndice C Avaliação dos Resultados do Problema de Planejamento da Operação (Complementação) 


\section{Lista de figuras}

Figura 1: Processo de decisão para um sistema hidrotérmico 24

Figura 2: Custos Imediato e Futuro X Armazenamento 33

Figura 3: Uso ótimo da água 34

Figura 4: Horizontes do Planejamento da Operação e Principais

Decisões em cada Estágio 36

Figura 5: Representação de Incertezas e do Detalhamento do Sistema com o Horizonte de Estudo $\quad 37$

Figura 6: Cadeia de Modelos Computacionais para o Planejamento da Expansão e Operação Energética 39

Figura 7: Exemplo de árvore de cenários (Árvore Completa) 44

Figura 8: Exemplo de sub-árvore de cenários do NEWAVE 45

Figura 9: Exemplo CEPEL - árvore completa 46

Figura 10: Exemplo CEPEL - sub-árvores $\quad 47$

Figura 11: Opção Atual $\quad 49$

Figura 12: Sorteio Condicionado $\quad 49$

Figura 13: Opção 1AAS $\quad 50$

Figura 14: Relação Demanda x Risco em um Ano Qualquer 52

Figura 15: Vazão Afluente Anual x Anos 53

Figura 16: Tendência Hidrológica $\quad 62$

Figura 17: Geração não condicionada - Primeira Etapa 62

Figura 18: Geração não condicionada - Segunda Etapa 63

Figura 19: Geração em Paralelo (Pente) para a simulação Forward 63

Figura 20: Seqüência de geração para a simulação Backward 64

Figura 21: Geração em Paralelo (Pente) para a simulação Backward 64

Figura 22: Seqüência Negativa $\quad 67$

Figura 23: Volume de regularização (Déficit) 69

Figura 24: Aplicação técnicas de agregação $\quad 77$

Figura 25: Exemplo ilustrativo do processo aglomerativo 78

Figura 26: Dendograma $\quad 79$

Figura 27: Divisão em 5 intervalos do domínio de duas VA (a)

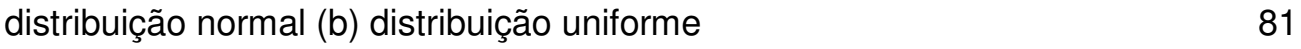

Figura 28: Valores sorteados para cada variável 82

Figura 29: Representação bi-dimensional de uma possível 
amostragem por hipercubo latino

Figura 30: Representação da amostragem utilizando método

Quase-Monte Carlo (Sobol) (a) 100 pontos (b) 1000 pontos

Figura 31: Amostra (a) Seqüência de Sobol

(b) Amostragem Aleatória Simples 86

Figura 32: Amostra (a) Seqüência de Sobol [50] (b) Seqüência de Halton [20] 87

Figura 33: Aplicação do Procedimento de Agregação 89

Figura 34: Escolha do Objeto Representativo 89

Figura 35: Probabilidade dos Cenários Forward 91

Figura 36: Opção $0 \quad 91$

Figura 37: Opção $1 \quad 92$

Figura 38: Opção 2

Figura 39: Opção $3 \quad 93$

Figura 40: Opção 4

Figura 41: Construção da FCF - modelo NEWAVE 95

Figura 42: Árvore Completa - Exemplo $\quad 97$

Figura 43: Sub-árvore (1‥ amostra) - Exemplo 97

Figura 44: - Sub-árvore - Exemplo (a) 2ํ․ amostra e (b) 3‥ Amostra 98

Figura 45: Sub-árvore após diversas reamostragens - Exemplo 98

Figura 46: Tendência Hidrológica Recente (\%MLT) 102

Figura 47: Estimativa Média (amostra de ruídos) 103

Figura 48: Estimativa Desvio-Padrão (amostra de ruídos) 104

Figura 49: Número de Rejeições (amostra de ruídos) 104

Figura 50: Intervalos da Distribuição Univariada 105

Figura 51: Exemplificação Classe $34 \quad 105$

Figura 52: Distribuição Multivariada - Amostra com Tamanho 2 mil a 100 mil 107

Figura 53: Estatística t - Média - Amostra Forward (ruído) 108

Figura 54: Estatística t - Desvio-padrão - Amostra Forward (ruído) 109

Figura 55: Teste de Aderência - Amostra Forward (ruído) 110

Figura 56: Distribuição Multivariada - Amostra Forward (ruído) 111

Figura 57: Estatística t - Desvio-padrãoMédia - Amostra Backward (ruído) 112

Figura 58: Estatística t - Desvio-padrão - Amostra Backward (ruído) 112

Figura 59: Teste de Aderência - Amostra Backward (ruído) 113

Figura 60: Distribuição Multivariada - Amostra Backward (ruído) 114

Figura 61: Estatística t - Média - Amostra Forward (ruído) -

Variando Tamanho Amostra Forward 
Figura 62: - Estatística t - Desvio-Padrão - Amostra Forward (ruído) Variando Tamanho Amostra Forward

Figura 63: Distribuição - Amostra Forward (ruído) - 250×20 - Opção Atual 116

Figura 64: Distribuição - Amostra Forward (ruído) - 300×20 - Opção Atual 116

Figura 65: Distribuição - Amostra Forward (ruído) - 250x20 - Opção 0116

Figura 66: Distribuição - Amostra Forward (ruído) - 300x20 - Opção 0117

Figura 67: Distribuição - Amostra Forward (ruído) - 250x20 - Opção 4117

Figura 68: Distribuição - Amostra Forward (ruído) - 300x20 - Opção 4117

Figura 69: Estatística t-Média - Amostra Backward (ruído) -

Variando Tamanho Amostra Backward

Figura 70: Estatística t -Desvio-Padrão - Amostra Backward (ruído) -

Variando Tamanho Amostra Backward

Figura 71: Estatística t-Média - Amostra Forward (ruído) -

Variando Tamanho Amostra Backward

Figura 72: Estatística t-Desvio-Padrão - Amostra Forward (ruído) -

Variando Tamanho Amostra Backward

Figura 73: Distribuição - Amostra Backward (ruído) - 200x50 - Opção Atual 121

Figura 74: Distribuição - Amostra Backward (ruído) - 200x100 - Opção Atual 121

Figura 75: Distribuição - Amostra Backward (ruído) - 200x50 - Opção 0 e 4121

Figura 76: Distribuição - Amostra Backward (ruído) - 200x100 - Opção 0 e 4122

Figura 77: Estatística t - Média - Amostra Forward (ruído) -

Variando Método Amostragem

Figura 78: Estatística t - DP - Amostra Forward (ruído) -

Variando Método Amostragem

Figura 79: Estatística t - Média - Amostra Backward (ruído) -

Variando Método Amostragem

Figura 80: Estatística t - DP - Amostra Backward (ruído) -

Variando Método Amostragem

Figura 81: Distribuição - Amostra Forward (ruído) - LHC 124

Figura 82: Distribuição - Amostra Backward (ruído) - LHC 125

Figura 83: Distribuição - Amostra Forward (ruído) - AD 125

Figura 84: Distribuição - Amostra Backward (ruído) - AD 125

Figura 85: Envoltória Cenários Forward - Sudeste 127

Figura 86: Envoltória Cenários Forward - Sul 128

Figura 87: Envoltória Cenários Forward - Nordeste 129

Figura 88: Envoltória Cenários Forward - Norte 130 
Figura 89: Média Cenários Forward - Sudeste 132

Figura 90: Desvio-Padrão Cenários Forward - Sudeste 134

Figura 91: Teste de Aderência Cenários Forward - Sudeste 135

Figura 92: Teste de Aderência Cenários Forward - Sul 136

Figura 93: Teste de Aderência Cenários Forward - Nordeste 137

Figura 94: Teste de Aderência Cenários Forward - Norte 137

Figura 95: Correlação Cruzada Cenários Forward - Sudeste x Sul 138

Figura 96: Correlação Cruzada Cenários Forward - Sudeste x Nordeste 139

Figura 97: Correlação Cruzada Cenários Forward - Sudeste x Norte 140

Figura 98: Correlação Cruzada Cenários Forward - Sul x Nordeste 141

Figura 99: Correlação Cruzada Cenários Forward -Sul x Norte 142

Figura 100: Correlação Cruzada Cenários Forward - Nordeste x Norte 143

Figura 101: Teste com Diferentes Níveis de Regularização - Opção Atual 145

Figura 102: Teste com Diferentes Níveis de Regularização - Opção $0 \quad 148$

Figura 103: Teste com Diferentes Níveis de Regularização -

$\begin{array}{lll}\text { Opções } 1 \mathrm{e} & 148\end{array}$

Figura 104: Teste com Diferentes Níveis de Regularização -

Opções 2, $\quad 3$ e 4

Figura 105: Amostra utilizada no teste não condicionado 150

Figura 106: Amostras utilizadas no teste condicionado 150

Figura 107: Envoltória Cenários Forward - Sudeste 151

Figura 108: Envoltória Cenários Backward - Sul 153

Figura 109: - Envoltória Cenários Backward - Nordeste 154

Figura 110: Envoltória Cenários Backward - Norte 155

Figura 111: Média Cenários Backward - Sudeste 157

Figura 112: Teste de Aderência Cenários Backward - Sudeste 158

Figura 113: Teste de Aderência Cenários Backward - Sul 160

Figura 114: Teste de Aderência Cenários Backward - Nordeste 161

Figura 115: Teste de Aderência Cenários Backward - Norte 162

Figura 116: Estatística t Média e Desvio-Padrão -

$\begin{array}{lll}\text { Cenários Backward - } \quad \text { Sudeste } & 164\end{array}$

Figura 117: Correlação Cruzada Cenários Backward - Sudeste x Sul 166

Figura 118: CC Backward x Forward - Sudeste x Sul - Opção Atual 167

Figura 119: CC Backward x Forward - Sudeste x Sul - Opção 0

Figura 120: CC Backward x Forward - Sudeste x Sul - Opção 1

Figura 121: CC Backward x Forward - Sudeste x Sul - Opção 4 
Figura 122: CC Backward x Forward - Sudeste x Sul - Opção 1AAS 169

Figura 123: CC Backward x Forward - Sudeste x Sul - Opção LHC 169

Figura 124: CC Backward x Forward - Sudeste x Sul - Opção AD 170

Figura 125: Comparação entre CC Forward e Backward 172

Figura 126: Diagrama esquemático das usinas hidroelétricas do

SIN 2008-2012 174

Figura 127: Representação dos subsistemas equivalentes 175

Figura 128: Convergência - Última Iteração 178

Figura 129: Convergência - Última Iteração - Variação Amostra - FEV/07 179

Figura 130: Trajetória Convergência - Variação Amostra -

$\begin{array}{ll}\text { Opção Atual - FEV/07 } & 180\end{array}$

Figura 131: Trajetória Convergência - Variação Amostra -

Opção 4 - FEV/07

Figura 132: Convergência - Última Iteração -

Variação Amostra - Com Reamostragem - FEV/07 182

Figura 133: CMO - Variação Amostra - Com Reamostragem - FEV/07 182

Figura 134: COPER - Variação Amostra - Com Reamostragem - FEV/07 183

Figura 135: Risco - Variação Amostra - Com Reamostragem - FEV/07 183

Figura 136: EENS - Variação Amostra - Com Reamostragem - FEV/07 183

Figura 137: Convergência - Última Iteração -

Variação Backward - Com Reamostragem - FEV/07 184

Figura 138: COPER - Variação Backward - Com Reamostragem - FEV/07 185

Figura 139: CMO - Variação Backward - Com Reamostragem - FEV/07 185

Figura 140: Risco - Variação Backward - Com Reamostragem - FEV/07 185

Figura 141: EENS - Variação Backward - Com Reamostragem - FEV/07 186

Figura 142: Convergência - Última Iteração -

Variação Forward - Com Reamostragem - FEV/07

Figura 143: Resultados - Variação Forward - Com Reamostragem - FEV/07 187

Figura 144: Convergência - Última Iteração -

Variação Forward \& Amostra - Opção Atual - FEV/07

Figura 145: Resultados - Variação Forward \& Amostra -

Opção Atual - FEV/07

Figura 146: Convergência - Última Iteração -

Variação Forward \& Amostra - Opção 0 - FEV/07

Figura 147: Resultados - Variação Forward \& Amostra - Opção 0 - FEV/07 189

Figura 148: Convergência - Última Iteração - 
Variação Forward \& Amostra - Opção 4 - FEV/07

Figura 149: Resultados - Variação Forward \& Amostra - Opção 4 - FEV/07 190

Figura 150: Convergência - Última Iteração -

Variação Forward \& Amostra - Opção 4 - FEV/07

Figura 151: COPER - Variação Tamanho da Amostra - FEV/07

Figura 152: CMO - Variação Tamanho da Amostra - FEV/07

Figura 153: Risco - Variação Tamanho da Amostra - FEV/07

Figura 154: EENS - Variação Tamanho da Amostra - FEV/07

Figura 155: Convergência - Última Iteração -

Variação Método de Amostragem - FEV/07

Figura 156: Resultados- Variação Método de Amostragem - FEV/07

Figura 157: Convergência - Última Iteração -

Variação Método de Amostragem e Backward - FEV/07

Figura 158: Resultados- Variação Método de Amostragem e

Backward - FEV/07

Figura 159: Convergência - Última Iteração -

Variação Método de Amostragem e Forward - FEV/07

Figura 160: Resultados- Variação Método de Amostragem e

Forward - FEV/07

Figura 161: Opções com combinação de LHC com agregação

Figura 162: Convergência - Última Iteração - Variação Amostra Combinação LHC e Agregação - FEV/07

Figura 163: CMO - Variação Amostra - Combinação LHC e

Agregação - FEV/07

Figura 164: COPER - Variação Amostra - Combinação LHC e

Agregação - FEV/07

Figura 165: Risco - Variação Amostra - Combinação LHC e

Agregação - FEV/07

Figura 166: EENS - Variação Amostra - Combinação LHC e

Agregação - FEV/07

Figura 167: Convergência - Última Iteração - Variação Forward Combinação LHC e Agregação - FEV/07

Figura 168: Resultados - Variação Forward - Combinação LHC e

Agregação - FEV/07

Figura 169: Convergência - Última Iteração - Variação Backward - 
Figura 170: Resultados - Variação Backward - Combinação LHC e Agregação - FEV/07

Figura 171: Resultados 45a iteração - Opção Atual 206

Figura 172: Resultados 45a iteração - Opção 0

Figura 173: Resultados 45a iteração - Opção 4

Figura 174: Resultados 45a iteração - Opção 0 com reamostragem 210

Figura 175: Resultados 45a iteração - Opção 4 com reamostragem 212

Figura 176: Esquema de relacionamento entre os módulos do programa NEWAVE

Figura 177: Cálculo da Função de Custo Futuro através da

Programação Dinâmica

Figura 178: Média Cenários Forward - Sul

Figura 179: Média Cenários Forward - Nordeste

Figura 180: Média Cenários Forward - Norte 245

Figura 181: Desvio-Padrão Cenários Forward - Sul 247

Figura 182: Desvio-Padrão Cenários Forward - Nordeste 248

Figura 183: Desvio-Padrão Cenários Forward - Norte 249

Figura 184: Média Cenários Backward - Sul 251

Figura 185: Média Cenários Backward - Nordeste 253

Figura 186: Média Cenários Backward - Norte 254

Figura 187: Estatística t Média e Desvio-Padrão -

Cenários Backward - Sul 256

Figura 188: Estatística t Média e Desvio-Padrão -

Cenários Backward - Nordeste

Figura 189: Estatística t Média e Desvio-Padrão -

Cenários Backward - Norte 259

Figura 190: Correlação Cruzada Cenários Backward - Sudeste x Nordeste 261

Figura 191: Correlação Cruzada Cenários Backward - Sudeste x Norte 263

Figura 192: Correlação Cruzada Cenários Backward - Sul x Nordeste 264

Figura 193: Correlação Cruzada Cenários Backward -Sul x Norte 266

Figura 194: Correlação Cruzada Cenários Backward - Nordeste x Norte 268

Figura 195:Resultados- Variação Backward - PMO Mai/04 269

Figura 196: Resultados- Variação Backward - PMO Set/04 270

Figura 197: Resultados- Variação Backward - PMO Set/09 270

Figura 198:Resultados- Variação Backward - PMO Jun/06 271

Figura 199:Resultados- Variação Backward - PMO Jul/06 272 
Figura 200Resultados- Variação :Backward - PMO Jan/07 272

Figura 201:Resultados- Variação Backward - PMO Fev/07 273

Figura 202:Resultados- Variação Backward - PMO Mai/07 273

Figura 203:Resultados- Variação Backward - PMO Jul/07 274

Figura 204:Resultados- Variação Backward - PMO Ago/07 275

Figura 205:Resultados- Variação Backward - PMO Jan/08 275

Figura 206:Resultados- Variação Forward - PMO Mai/04 276

Figura 207:Resultados- Variação Forward - PMO Set/04 276

Figura 208:Resultados- Variação Forward - PMO Set/09 277

Figura 209:Resultados- Variação Forward - PMO Jun/06 278

Figura 210:Resultados- Variação Forward - PMO Jul/06 278

Figura 211:Resultados- Variação Forward - PMO Jan/07 279

Figura 212:Resultados- Variação Forward - PMO Fev/07 279

Figura 213:Resultados- Variação Forward - PMO Mai/07 280

Figura 214:Resultados- Variação Forward - PMO Jul/07 281

Figura 215:Resultados- Variação Forward - PMO Ago/07 281

Figura 216:Resultados- Variação Forward - PMO Jan/08 282

Figura 217:Resultados- Variação Semente - Opção 4 - PMO Set/05 282

Figura 218:Resultados- Variação Semente - PMO Set/04 283

Figura 219:Resultados- Variação Semente - PMO Jul/07 284

Figura 220:Resultados- Variação Semente - PMO Jan/08 284

Figura 221:Resultados- Variação Amostra \& Semente -

Opção 4 - PMO Jan/08 285

Figura 222:Resultados- Variação Forward \& Semente -

Opção 4 - PMO Set/04

Figura 223:Resultados- Variação Forward \& Semente -

Opção 4 - PMO Jul/07 286

Figura 224:Resultados- Variação Forward \& Semente -

Opção 4 - PMO Jan/08 287

Figura 225:Resultados- Variação Forward \& Amostra -

Opção 4 - PMO Jul/07 287

Figura 226:Resultados- Variação Forward \& Amostra -

Opção 4 - PMO Jan/08

Figura 227:Resultados- Variação Semente -

Opção 4 \& Variantes - PMO Set/04

Figura 228:Resultados- Variação Amostra - 
Opção 4 \& Variantes - PMO Jan/08

Figura 229:Resultados- Variação Backward -

Opção 4 \& Variantes - PMO Set/04

Figura 230:Resultados- Variação Backward -

Opção 4 \& Variantes - PMO Jul/06

290

Figura 231:Resultados- Variação Backward -

Opção 4 \& Variantes - PMO Jan/08

Figura 232:Resultados- Variação Forward -

Opção 4 \& Variantes - PMO Set/04

Figura 233:Resultados- Variação Forward -

Opção 4 \& Variantes - PMO Jul/06

Figura 234:Resultados- Variação Forward -

Opção 4 \& Variantes - PMO Jan/08

293 


\section{Lista de tabelas}

Tabela 1: Valores Padrão para IC 95\% 73

Tabela 2: Correlação Cruzada Média Cenários Forward 143

Tabela 3: Análise das Seqüências Negativas - Opção Atual 144

Tabela 4: Teste de Máximos - Opção Atual 145

Tabela 5: Análise das Seqüências Negativas - Opção 0

Tabela 6: Análise das Seqüências Negativas - Opções 1 e 1AAS 146

Tabela 7: Análise das Seqüências Negativas - Opções 2, 3 e 4

Tabela 8: Teste de Máximos - Opção 0

Tabela 9: Teste de Máximos - Opções 1 e 1AAS 147

Tabela 10: Teste de Máximos - Opções 2, 3 e 4

Tabela 11: Correlação Cruzada Média Cenários Backward -

$\begin{array}{ll}\text { Não Condicionado } & 170\end{array}$

Tabela 12: Número de iterações para convergência 177

Tabela 13: Número de iterações para convergência (cont.) 177 


\section{Lista de siglas e abreviaturas}

$A D$ - amostragem descritiva

AAS - amostragem aleatória simples

CCEE - Câmara de Comercialização de Energia Elétrica

Cepel - Centro de Pesquisas de Energia Elétrica

$\mathrm{CMO}$ - custo marginal de operação

COPER - valor esperado do custo total de operação para a simulação final

EENS - valor esperado da energia não suprida

ENA - energia natural afluente

EPE - Empresa de Pesquisa Energética

FCF - função de custo futuro

$\mathrm{FCl}$ - função de custo imediato

LHC - hipercubo latino (sigla em inglês)

MME - Ministério de Minas e Energia

NLEQ - número de cenários backward (aberturas)

NSIM - número de cenários forward

ONS - Operador Nacional do Sistema Elétrico

$\operatorname{PAR}(p)$ - modelo autorregressivo periódico de ordem $p$ (sigla em inglês)

PDD - programação dinâmica determinística

PDDE - programação dinâmica dual estocástica ( em inglês SDDP)

PDE - programação dinâmica estocástica

PMO - programa mensal de operação

SIN - sistema interligado nacional

ZINF - limite inferior do valor esperado do custo total de operação

ZSUP - limite superior do valor esperado do custo total de operação 